\title{
COLLAGEN CROSSLINKING FOR KERATOCONUS CAN CHANGE SCLERAL SHAPE
}

Gregory DeNaeyer OD ${ }^{1}$ and Donald R Sanders MD, $\mathrm{PhD}^{2}$

${ }^{1}$ Optometrist at Arena Eye Surgeons

${ }^{2}$ Director, Center for Clinical Research and President and CEO, Visionary Optics LLC

Corresponding Author Donald Sanders MD, PhD: drsmd@drsmd.com

Submitted: November 27, 2017. Accepted: March 3, 2018. Published: March 23, 2018.

\begin{abstract}
Collagen crosslinking (CXL) for keratoconus is known to decrease, halt, or even partially reverse progression of keratoconus. We report on a case where a substantial effect on scleral shape was also demonstrated. This keratoconus patient, who was successfully wearing a scleral lens, underwent collagen crosslinking OS. Three-months post crosslinking, the patient was unable to wear his previous scleral lens due to lens discomfort. Elevation mapping pre and post crosslinking, with a new corneal-scleral topography system, showed a decrease in size and change in shape of the cone, as well as a substantial change in the scleral elevation pattern at a 16-mm chord diameter. The change in the scleral elevation pattern was best observed on scleral shape plots which graph the sagittal height (SAG) value on the Y-axis vs. meridian on the $\mathrm{X}$-axis. The pre-crosslinking plot resembled a standard toric curve although the depression inferiorly at $327^{\circ}$ was deeper than that superiorly by $>250 \mu$. The post-crosslinking plot changed substantially with the steep axis superiorly being markedly attenuated: the SAG superiorly $\left(106^{\circ}\right)$ decreased post-crosslinking by $>350 \mu$. Attempts to virtually fit the eye surface post-crosslinking with a standard posterior toric haptic demonstrated a good fit superiorly and a poor fit inferiorly. The post-crosslinking scleral toricity plot was used to obtain quantitative information to manufacture a custom design conforming to this specific eye. The resulting lens was comfortable, well centred and resulted in a BCVA of 20/30.
\end{abstract}

Collagen crosslinking (CXL) using riboflavin and UV-A light has been shown to slow, stop, and in some cases reverse the progression of keratoconus. ${ }^{1,2}$ It appears to biomechanically strengthen the cornea up to $300 \%$ through its effect on the collagen lamellae ${ }^{3}$ in a manner similar to that seen in the cornea with aging. ${ }^{4}$ While there are over 400 articles in the peer-reviewed literature on corneal collagen crosslinking (PubMED, accessed 10/24/17 “corneal collagen crosslinking”), to our knowledge there have been no clinical reports on the effects of the procedure on the sclera. This report documents one such case.

\section{CASE STUDY}

This 44-year-old male patient with bilateral keratoconus (OS $>\mathrm{OD})$ had been previously fit with a soft contact lens OS in 2014 (Hydrokone, Visionary Optics, Front Royal, Virginia) with a BCVA of 20/30. The 
keratoconus progressed in OS until in November 2016, the BCVA was 20/60 with the Hydrokone soft lens. The patient was measured with the sMap3D Cornealscleral topographer (Precision Ocular Metrology, Los Angeles, CA) for a scleral lens fitting, which predicted the need for a $2 \mathrm{D}$ toric peripheral haptic (the steps in how diopters of toricity were calculated have been reported in a previous publication. ${ }^{5}$ ) The left eye was refit with the Europa Scleral lens (Visionary Optics, Front Royal, VA) using the lens parameters predicted by the sMap3D, which demonstrated a good, comfortable fit with a BCVA of 20/25. Significant steepening was noted from topography taken Oct 2016 compared to topography of Oct 2014 which correlated with loss of visual acuity. Crosslinking was recommended secondary to progression. Because of the history of keratoconus progression, the left eye underwent a CXL procedure in January 2017. By April 2017, the patient had restarted wear of the scleral lens but reported discomfort, redness, and difficulty removing the lens; the sMap3D examination was repeated. Figure 1 shows the pre and post crosslinking composite corneal and scleral elevation maps. The close-up inferior insets demonstrate a shrinking of the cone with CXL.
A change in the scleral elevation pattern is best observed on scleral shape plots ${ }^{6}$ which are derived from the sagittal height (SAG) data on the corneal and scleral elevation maps; sagittal height at any point or any chord diameter from the corneal center can be directly calculated from the measured corneal and scleral elevation topography. Figure 2A demonstrates a scleral elevation map taken with the sMap3D of a case with 6.5 diopters of scleral toricity at a diameter of $16 \mathrm{~mm}$ around the center of the cornea. The steep axis is an area of depression (blue colour) on the surface. Figure 2B compares the scleral elevation map which is a visual qualitative assessment of scleral shape to the circumferential scleral shape plot which is a quantitative assessment. This graph plots the axis in degrees on the X-axis and the SAG value of the eye on the Y-axis (larger values as one goes down the scale) circumferentially at a radius of $8 \mathrm{~mm}$ (diameter of $16 \mathrm{~mm}$ ) from the corneal center. Measuring circumferentially, in a case with regular toricity, an area of depression alternates with an area of elevation every $90^{\circ}$. This describes a toric $\left(\mathrm{Sin}^{2}\right)$ curve with a periodicity of $180^{\circ}$. In this particular case, the steep axes superiorly and inferiorly are

FIG. 1 Composite corneal and scleral elevation maps pre CXL (A) and post CXL (B). The colour scale (centre) is the same for both maps.
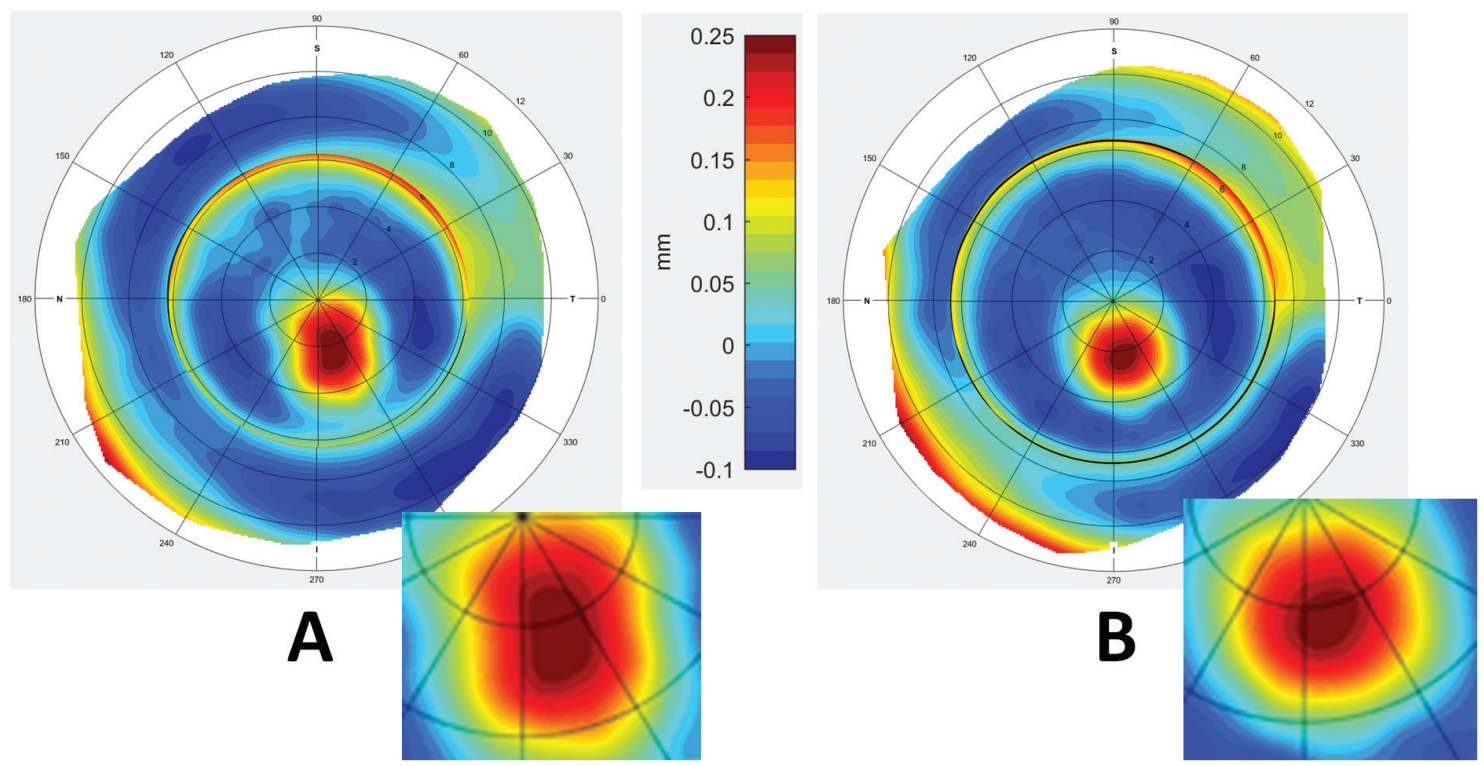

J Cont Lens Res Sci Vol 2(1):e15-e21; March 23, 2018

This article is distributed under the terms of the Creative Commons Attribution-Non Commercial 4.0 International License. 
represented by the low areas on the toricity plot and the flat axes are represented by the high areas. Note that each scale point on the Y-Axis is $0.2 \mathrm{~mm}(200 \mu)$ and from the highest to lowest point on the graph, the change in SAG is $>1 \mathrm{~mm}(>1,000 \mu)$.

Figure 3 demonstrates the scleral shape plots pre (A) and post (B) CXL graphed on the same scale. The
post-CXL graph appeared to have at least $100 \mu$ less SAG in all meridians; the SAG superiorly $\left(105.5^{\circ}\right)$ decreased post-CXL by $>350 \mu$. The pre-crosslinking plot resembled more a standard toric peripheral curve (largely conforming to a $\mathrm{Sin}^{2}$ curve with approximately $180^{\circ}$ periodicity ${ }^{6}$ ) although the depression inferiorly at $330^{\circ}$ was deeper than that superiorly by $250 \mu$. The

FIG. 2 Comparison of the scleral elevation map (A) to the scleral shape plot (B) in a case with high and fairly regular scleral toricity. The long arrows point to the same area of the sclera at a $16 \mathrm{~mm}$ diameter from the corneal centre.
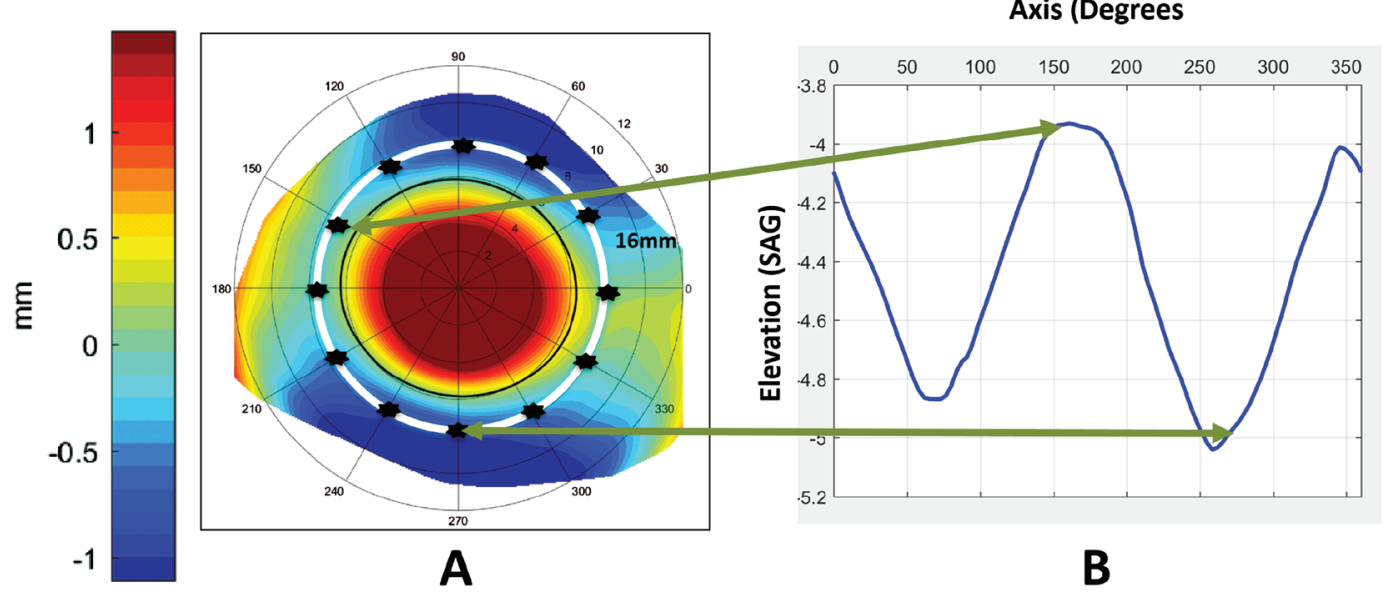

FIG. 3 Pre (A) and 3 months Post (B) CXL scleral shape plots at a 16-mm diameter. The X-axis is meridian in degrees and the Y-axis is sagittal height (SAG), graphed on the same scale in both plots. The mean SAG pre CXL across all axes was $4.480 \mathrm{~mm}(4480 \mu)$ and post CXL was $4.606 \mathrm{~mm}(4606 \mu)$, a difference of $126 \mu$.

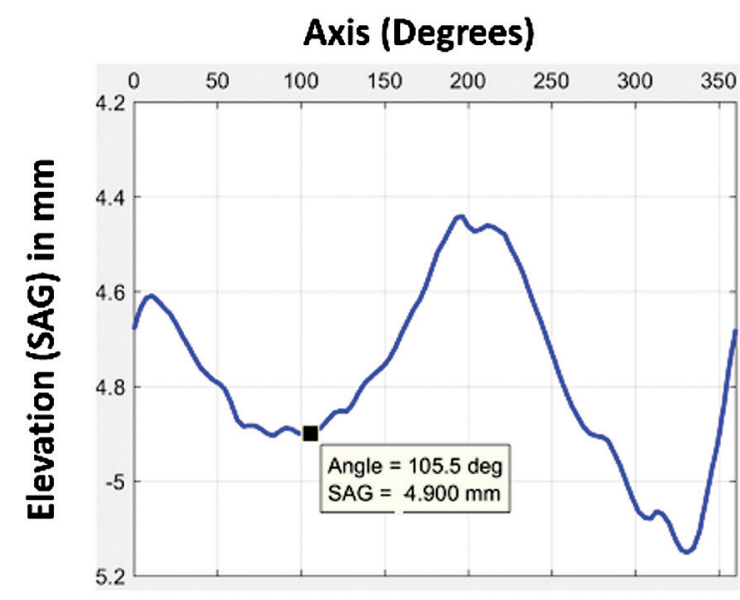

A

\section{Axis (Degrees)}

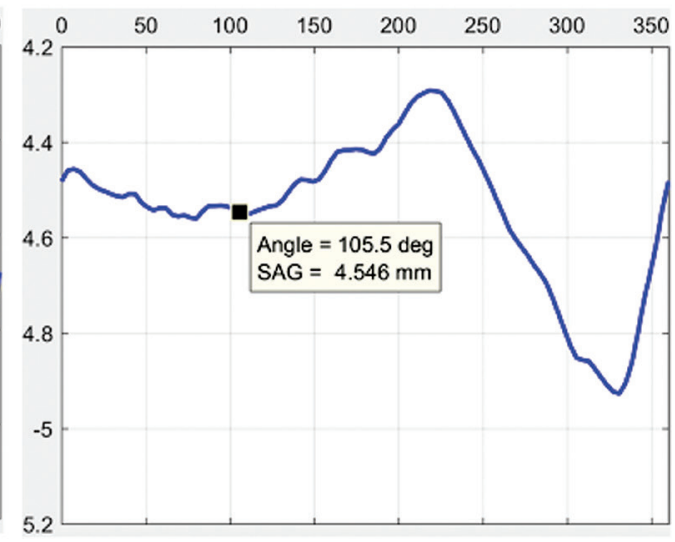

B

J Cont Lens Res Sci Vol 2(1):e15-e21; March 23, 2018

This article is distributed under the terms of the Creative Commons Attribution-Non Commercial 4.0 International License. 
post-crosslinking plot had changed substantially with the steep axis superior depression being markedly attenuated.

The difference between the steep axis of the sclera superiorly and inferiorly post CXL was obvious on the 3D image (Figure 4).

Attempts to virtually fit the post-crosslinking eye surface with the Europa scleral lens with a standard posterior toric haptic using the sMap3D software demonstrated a good fit superior-nasally and a poor fit (edge lift) inferior-temporally. This is demonstrated in the colour-coded 3D lens elevation map (Figure 5) and the simulated fluorescein map (Figure 6).

The post-crosslinking scleral shape plot was used to obtain quantitative information (Figure 7) to manufacture a custom (multi-meridian) design conforming to this specific eye. The resulting lens (Figure 8) was comfortable, well centred and resulted in a BCVA of 20/30.

\section{DISCUSSION}

Wollensak, Spoerl and Seiler ${ }^{1}$ first reported on the use of the CXL procedure to halt or reduce the progression of keratoconus in patients. Their work has been confirmed in multiple studies including in a recent randomized, prospective, double masked, sham controlled clinical trial ${ }^{2}$. In animal work, a significant increase in corneal biomechanical stiffness after the procedure was noted. ${ }^{3}$ More recent animal studies in rabbits have demonstrated a similar stiffening effect with CXL on sclera both in vitro ${ }^{7,8}$ and longer term, ${ }^{9}$ although in vitro studies on human sclera have had mixed results with one study showing no effect and

FIG. 4 Three-dimensional sagittal eye image in the superior-inferior plane demonstrating increased steepness inferiorly.

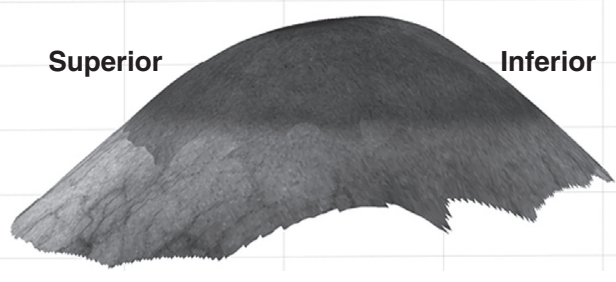

another study showing a stiffening effect. ${ }^{8,10}$ Selective areas of stiffening of the sclera based upon riboflavin accumulation and UV light exposure could explain scleral shape changes observed. Alternately, it is possible that the observed changes are due crosslinking effects on the conjunctiva or even transmitted stress or tightening from the cornea; there is insufficient information to rule out any of these mechanisms. Regardless of the mechanism, it is not surprising that CXL can change scleral shape, however, up until recently, the means to measure scleral shape was not readily available clinically.

The patient was 44 and typically most $\mathrm{KC}$ patients have plateaued in their 40s (the cornea crosslinks naturally with age). Without change these patients aren't really candidates for crosslinking. However, in this case, the patient progressed and the standard of care is to have those patients evaluated and treated accordingly.

Could these observed differences in pre to post CXL measurements be simply due to measurement variability? A number of factors mitigate against this possibility. Firstly, some sort of change undoubtedly occurred on the ocular surface to explain why a well-fitting scleral lens, fit using the sMap3D fitting algorithm, no longer fit on the eye post CXL 3 months later. Secondly, the post CXL surface data was used to modify the fit in a highly customized fashion and was highly successful. Thirdly, the changes observed pre and post CXL are outside the range of variability observed in sMap3D repeatability studies. The repeatability of measurements with the sMap3D instrument has been reported in 2 series of cases; 19 normal eyes ${ }^{11}$ and 25 eyes scheduled for scleral lens fitting. ${ }^{5}$ The mean difference in SAG between the 2 exams in the first series was $6 \mu$ with a standard deviation of $40 \mu$. The SAG difference between exams was $\pm 25 \mu$ in $39 \%$ of cases, $\pm 50 \mu$ in $78 \%$ of cases, $\pm 75 \mu$ in $94 \%$ of cases and all cases within $\pm 100 \mu$. In the second series, the mean difference in SAG between the 2 examinations was $14 \mu$ with a SD of $55 \mu$. Sixty-eight percent of cases had a difference of $\leq 50 \mu$, and $95 \%$ of cases had a difference of $\leq 100 \mu$. The change in SAG, pre to post CXL, was $126 \mu$ which was outside the range of mean \pm 2 standard deviations in both series

J Cont Lens Res Sci Vol 2(1):e15-e21; March 23, 2018

This article is distributed under the terms of the Creative Commons Attribution-Non Commercial 4.0 International License. 
FIG. 5 Colour-coded 3D lens elevation map of the fit of the post-crosslinking eye surface with a standard toric posterior haptic demonstrating edge lift inferior-temporally. The front surface of the eye is demonstrated by a colour-coded clearance map. The scale to the left demonstrates the predicted clearance of the lens in mm. The blue colours represent clearance between the ocular surface and the fitted scleral lens. Green and warmer colours represent various degrees of touch. The purple represents the back surface of the fitted scleral lens. $\mathrm{N}=$ nasal, $\mathrm{I}=$ inferior, $\mathrm{T}=$ temporal. The edge lift noted on the right side of the image is demonstrated both by the distance between the colour-coded ocular surface and the purple scleral lens but also by the colour of the ocular surface (blue rather than yellow-orange).
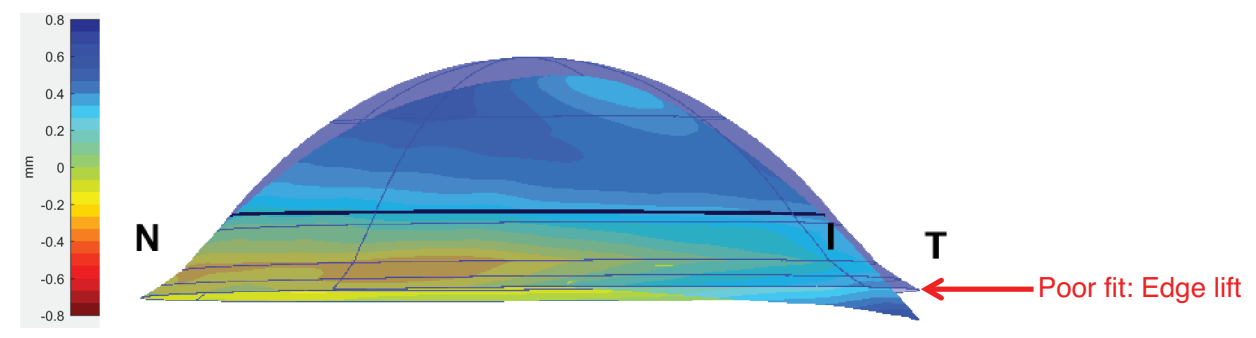

FIG. 6 Simulated fluorescein pattern of the fit of the post-crosslinking eye surface with a scleral lens with a standard toric posterior haptic demonstrating good peripheral fit in 3 quadrants and edge lift inferiortemporally. Right: anterior-posterior view with polar axis. Left: sagittal image.
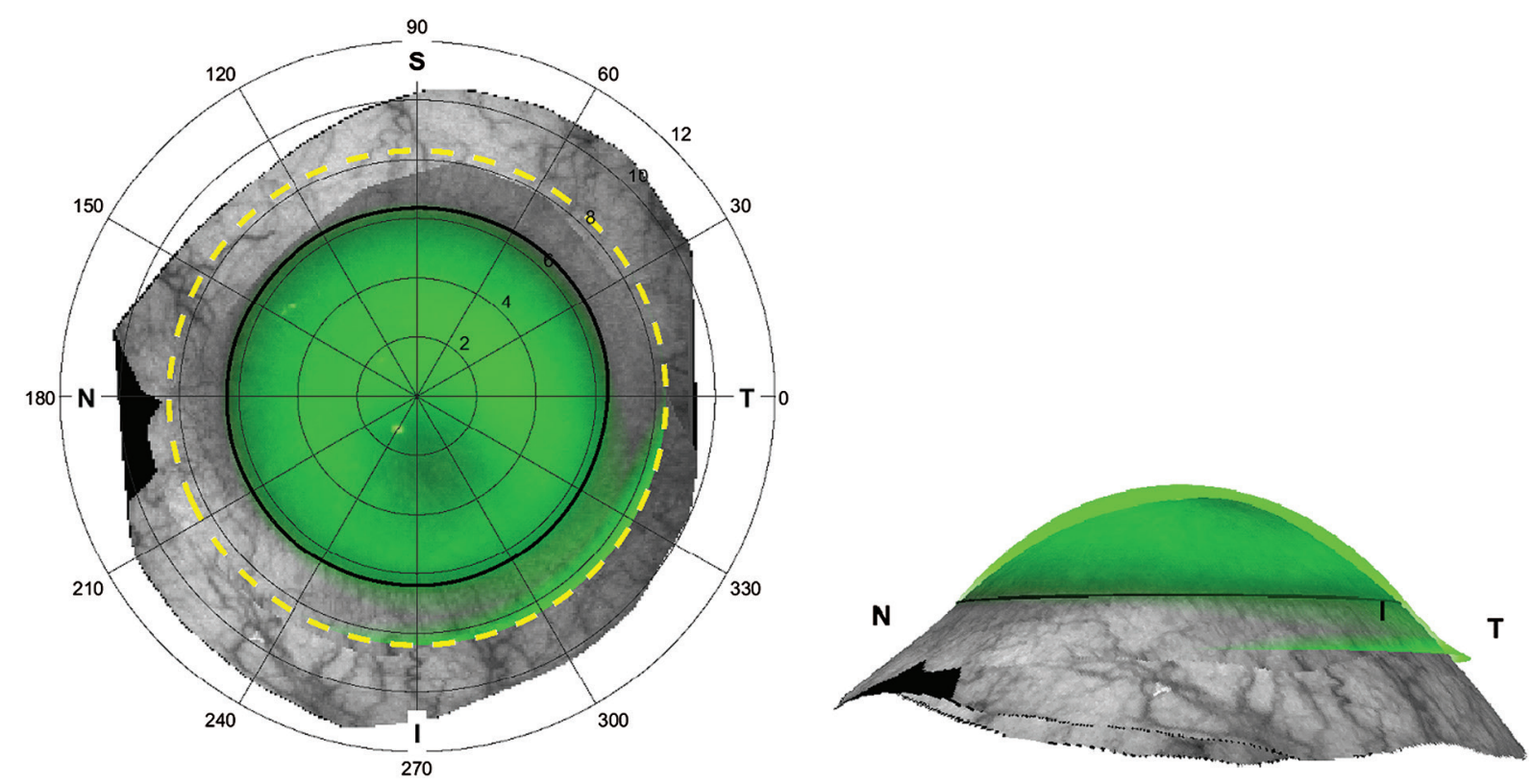

J Cont Lens Res Sci Vol 2(1):e15-e21; March 23, 2018

This article is distributed under the terms of the Creative Commons Attribution-Non Commercial 4.0 International License. 
FIG. 7 Post-crosslinking scleral shape plot at a $16 \mathrm{~mm}$ diameter used to design a custom back surface scleral lens. These 4 data points and similar data from $14 \mathrm{~mm}$ and $15 \mathrm{~mm}$ diameter plots were programmed into the scleral lens manufacturing lathe software to produce this lens custom peripheral back surface.

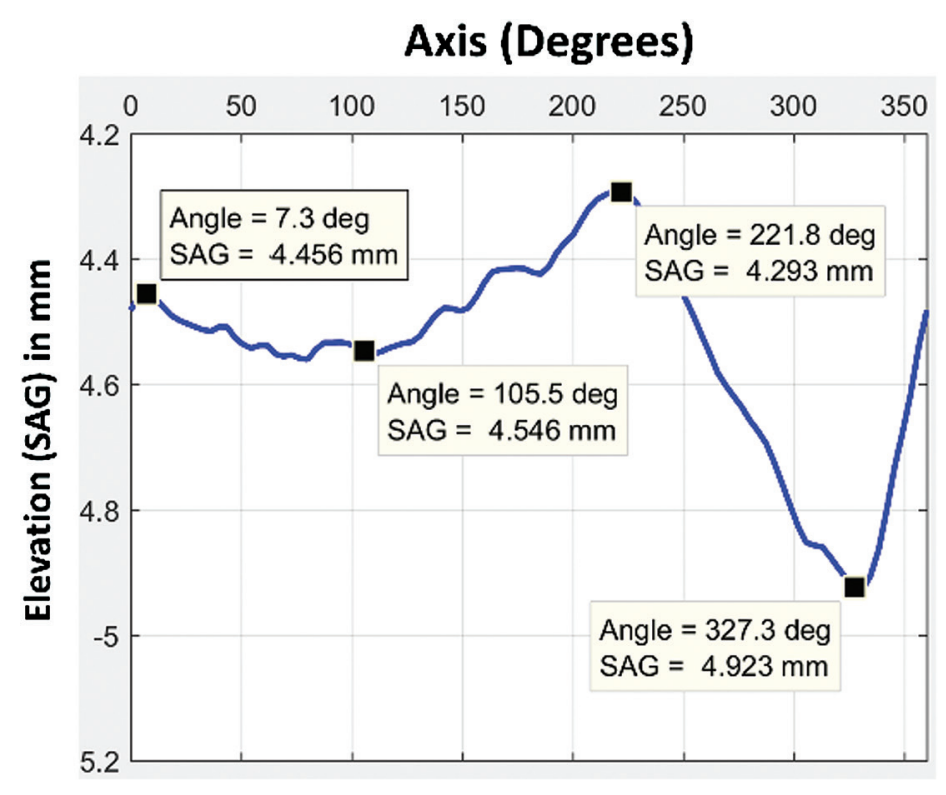

FIG. 8 Post-crosslinking eye fit with a custom back surface scleral lens based upon scleral shape plots. The lens was well centred with a comfortable fit.

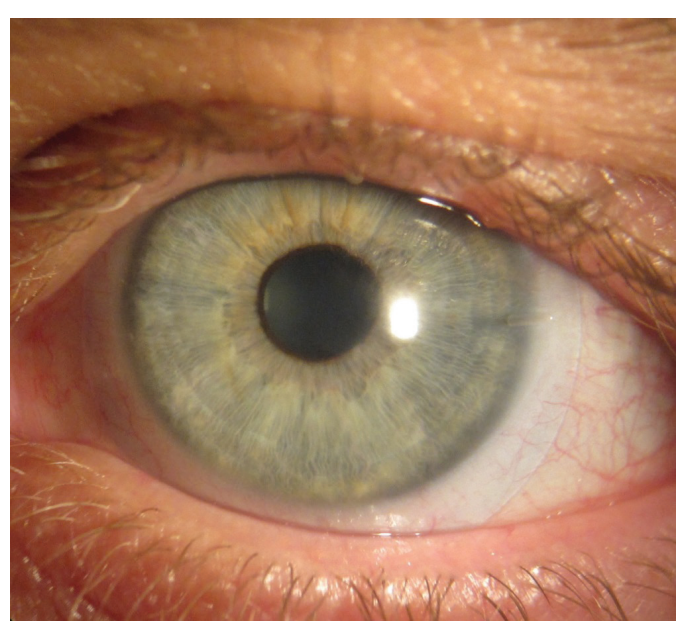


making it highly unlikely that these findings are due to measurement variability.

Differences in centration of the cornea between topography examinations can effect mapping measurements however the sMap3D ameliorates this issue. Integral to the mapping and stitching of the images in a single examination is the identification of the limbus; this is done automatically by the software but can be over-ridden by the user in complex cases. By using the limbal position as a fixed point in both pre and post CXL exams, it is highly unlikely that centration differences were a factor.

This patient, wearing a well-fitting scleral lens for 2 months, underwent a CXL procedure. Three months post CXL, the lens no long fit the eye. Because corneal-scleral topography was done pre and post CXL, the changes induced by the procedure could be documented. The sMap3D instrument was able to provide quantitative data sufficient to design a well-fitting custom back surface scleral lens. With the growing use of the CXL procedure, the scleral lens fitter should be aware of a possible change in the scleral shape and monitor these patients closely.

\section{DISCLOSURE}

Drs. DeNaeyer and Sanders are shareholders in Precision Ocular Metrology, the manufacturer of the sMap3D instrument. Dr. Sanders is a shareholder of Visionary Optics and Dr. DeNaeyer is a consultant to Visionary Optics. Visionary Optics distributes the sMap3D.

Visionary Optics helped fund this research.

\section{REFERENCES}

1. Wollensak G, Spoerl E, Seiler T. Riboflavin/ultravioletA induced collagen crosslinking for the treatment of keratoconus. Am J Ophthalmol 2003;135:620-27.

2. Hersh PS, Stulting RD, Muller D, et.al. United States multicenter clinical trial of corneal collagen crosslinking for keratoconus treatment. Ophthalmology 2017 Oct;124(10):1475-84.

3. Wollensak G, Spoerl E, Seiler T. Stress-strain measurements of human and porcine corneas after riboflavinultraviolet-A induced cross-linking. J Cataract Refract Surg 2003;29:1780-85.

4. Cartwright NEK, Tyrer JR, Marshall J. Age-related differences in the elasticity of the human cornea. Invest Ophthalmol Vis Sci 2011;52:4324-29.

5. DeNaeyer GD, Sanders DR. sMap3D corneo-scleral topographer repeatability in scleral lens patients. Eye Cont Lens 2017 Jul 21. doi: [Epub ahead of print].

6. DeNaeyer G, Sanders DR van der Worp, E, et.al. Qualitative assessment of scleral shape patterns using a new wide field ocular surface elevation topographer: the SSSG study. J Cont Lens Res Sci 2017 Nov 1(1):1222; http://www.jclrs.org/index.php/JCLRS/article/view/11

7. Zhang Y, Zou C, Liu L, et.al. Effect of irradiation time on riboflavin-ultraviolet-A collagen crosslinking in rabbit sclera. J Cataract Refract Surg 2013 Aug;39(8):1184-9.

8. Zhang Y, Li Z, Liu L, et. al. Comparison of riboflavin/ ultraviolet-A cross-linking in porcine, rabbit, and human sclera. Biomed Res Int 2014;2014:194204. https:// www.ncbi.nlm.nih.gov/pmc/articles/PMC3910020/

9. Wollensak G, Iomdina E. Long-term biomechanical properties of rabbit sclera after collagen crosslinking using riboflavin and ultraviolet A (UVA). Acta Ophthalmol 2008 Dec;86(8):887-93 http://onlinelibrary. wiley.com/doi/10.1111/j.1755-3768.2008.01229.x/full

10. Wang M, Zhang F, Qian X, et.al. Regional Biomechanical properties of human sclera after cross-linking by riboflavin/ultraviolet A. J Refract Surg 2012 Oct;28(10):723-8.

11. Morrison, SD, Caroline, PJ, DeNaeyer G. et. al. Repeatability of SAG and scleral toricity values using a new corneo-scleral topography unit. GSLS Poster January 22, $2017 \mathrm{http}: / / w w w . g s l s y m p o s i u m . c o m / g e t a t t a c h m e n t /$ Posters/Morrison,-Sheila-Repeatability-of-SAG-andScleral-Toricity-Values.pdf.aspx. 